\title{
Article
}

\section{Systematic Understanding of the Mechanism of Pristimerin via Computational Target Fishing}

\author{
Zaiyu $\mathrm{Li}^{{ }^{*}}$, Cong $\mathrm{Hu}^{2 *}$, Yu zhen ${ }^{3}$, Bo Pang ${ }^{4}$, Huanfa $\mathrm{Yi}^{2}$, Xianglin Chen ${ }^{1 *}$ \\ ${ }^{1}$ Department of Neurosurgery, The Sixth Hospital Affiliated to Guangzhou Medical University, Guangzhou, \\ Guangdong, China; \\ 2 Central Llaboratory of the Eastern Division, The First Hospital of Jilin University, Changchun, Jilin, China; \\ 3 Department of Dermatology, The First Hospital of Jilin University, Changchun, Jilin, China; \\ 4 Department of Cardiology, The First Hospital of Jilin University, Changchun, Jilin, China; \\ anny.hc@163.com \\ lizaiyu666@aliyun.com \\ 13612611@qq.com \\ 64302486@qq.com \\ yihuanfa@jlu.edu.cn \\ *These authors contributed equally to this work. \\ \#Correspondence: gylynk@hotmail.com; Tel.: +86-135-0432-4523
}

\begin{abstract}
Background:Pristimerin (PM) is a naturally occurring quinonemethide triterpenoid compound that isolated from the Celastraceae and Hippocrateaceae families. Its anticancer effects have attracted a great deal of attention, but the mechanisms of action remain obscure; 2) Methods: In this study, we screened for the active compounds of Pristimerin using a drug-likeness approach. Potential protein targets of Pristimerin were predicted by PharmMapper and Coremine database. Candidate protein targets were then uploaded to GeneMANIA and GO pathway analysis; 3) Results:Finally, compound-target, target-pathway, and compound-target-pathway networks were constructed using Cytoscape 3.3; and 4) Conclusions: The results showed that Pristimerin had good drug ability and identified 13 putative protein targets. Network analysis revealed that these targets are associated with cancer, inflammation and other physiological processes. In summary, Pristimerin is predicted to target a variety of proteins and pathways to form a network that exerts systemic pharmacological effects.
\end{abstract}

Dataset:

\section{Dataset License:}

Keywords: Pritimerin; target fishing; druggability; network pharmacology

\section{Introduction}

Pristimerin(20 $\alpha$-3-hydroxy-2-oxo-24-nor-friedela-1-10,3,5,7-tetraen-carboxylicacid-29-meth ylester, PM, Figure1A) is a naturally occurring quinonemethide triterpenoid compound, isolated from the family Celastraceae and Hippocrateaceae. Pristimerin is reported to have a variety of pharmacological effects such as anti-cancer, anti-angiogenic, anti-inflammatory and anti-protozoal, as well as insecticidal activities[1]. Its anti-tumor activities have already been confirmed on a series 
of human cancer cell lines such as colon cancer, breast cancer, prostate cancer, and multiple myeloma tumors[2-6], and the anti-tumor function was associated with various molecular targets [7]. A number of natural triterpenoid which are structurally related to pristimerin such as celastrol[8], ursolic acid[9] and betulinic acid[10]. Though the anticancer effects especially in cancer chemoprevention and treatment have attracted a great deal of attention[7], the mechanisms of action remained obscure.

Despite the good permeability, Pristimerin has reported to have a lower oral bioavailability $(\sim 28.4 \%)$ in rats [11]. Poor bioavailability is due to the relatively high efflux from the Pgp mechanism. Therefore, in the presence of verapamil, the oral bioavailability of Pristimerin is almost twice as high (1728.1 $\mu \mathrm{g} \mathrm{h/mL}$ when given with verapamil vs. AUC0-t of $949.2 \mu \mathrm{g} \mathrm{h} / \mathrm{mL}$ when given alone) in rats[12]. For these naturally occurring compounds, knowledge of toxicity or medicinal properties is usually earlier than an accurate understanding of the target or mechanism, and there is still a lot of knowledge about Pristimerin.

In this study, the TCMSP server was first used to evaluate the drug availability of Pristimerin. Potential targets were predicted by calculating reverse docking and chemical-protein interaction analysis, and overlapping targets identified using both methods were selected for further study based on gene ontology (GO) and pathway analysis. Finally, we constructed a drug target network to systematically outline the potential targets and mechanisms of action of Pristimerin. An overview of the experimental procedure for Pristimerin target prediction is shown in Figure 1B.
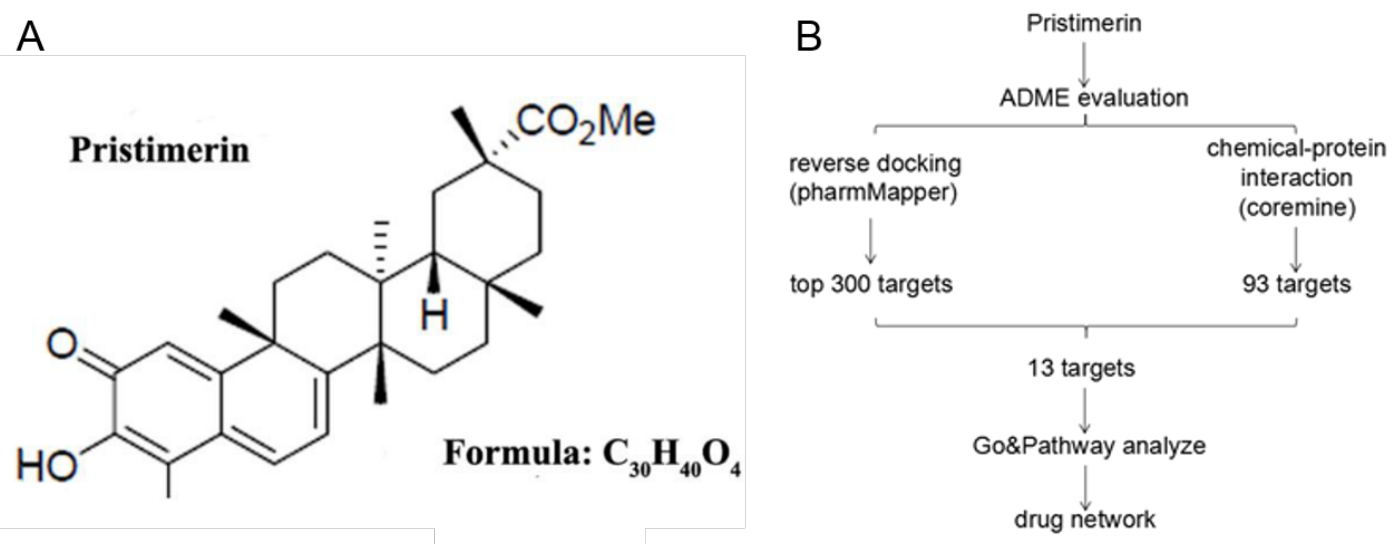

Figure 1. Chemical structure and Pipeline for the identification of Pristimerin. (A) Chemical structure of Pristimerin downloaded from the PubChem database (CID: 159516); (B) Pipeline for the identification of putative Pristimerin targets that integrates ADME evaluation, reverse docking, chemical-protein interaction, GO, and pathway analyses, and network construction.

\section{Materials and Methods}

2.1. Evaluation of Drug-Likeness The TCMSP server (http://ibts.hkbu.edu.hk/LSP/ tcmsp.php) is a system-level Chinese medicine (TCM) pharmacology database that can calculate ADME (absorption, distribution, metabolism and excretion), related properties to interesting, naturally occurring compounds[13]. It provides an evaluation model, which integrated oral bioavailability (OB), drug-likeness (DL), Caco-2 permeability and so on. Drug-like (DL) is a method of qualitatively estimating a "drug-like" compound that helps optimize pharmacokinetics and drug 
properties such as solubility and chemical stability. Building a database-dependent model based on molecular descriptors and Tanimoto coefficients (as displayed below) :

$$
\mathrm{T}(\mathrm{A}, \mathrm{B})=\frac{\mathrm{A} \times \mathrm{B}}{|| \mathrm{A}|| 2+|| \mathrm{B}|| 2-\mathrm{A} \times \mathrm{B}}
$$

where $\mathrm{A}$ is the molecular descriptor of herbal ingredients, and B shows the average molecular properties of all molecules in Drug-Bank database. The Oral bioavailability (OB) plays an important role in the efficiency of the drug delivery to the systemic circulation, which is one of the most vital pharmacokinetic properties of orally administered drugs. The OB value of all herbal ingredients was calculated using the internal model OBioavail 1.1. The Caco-2 cell line has been widely used to predict the oral absorption properties of drugs in the intestinal epithelial cell barrier, which also applies to the TCMSP database.

2.2 Computational Target Fishing by PharmMapper and Coremine database PharmMapper can identify potential protein targets for small molecule compounds by pharmacophore mapping [14]. It can provide the first 300 to 3000 targets for a given compound, sorted in descending order of appropriate scores. Meanwhile, the Coremine database can identify potential target proteins by chemical-protein interaction analysis of small molecules[15]. Both of them are powerful tools for computational target fishing. We entered Pristimerin (PubChem CID: 159526) to the PubChem and Coremine database, then downloaded an SDF file and uploaded it to the PharmMapper. All parameters were set to default values, and we chose the overlapping ones for further investigation.

2.3 Analysis by GeneMANIA GeneMANIA is a flexible, user-friendly web interface for generating hypotheses about gene function, analyzing gene lists and prioritizing genes for functional assays[16]. The results were exhibited after we input the target genes of interest in the previous step.

GO and Pathway Analysis, and Network The PANTHER (http://pantherdb.org), a comprehensive, curated database of protein families, trees, subfamilies and functions, can be used to help understand relationships in gene expression data and provide systematic and visual information on the gene of interest[17]. After uploaded the interested target genes following the online instructions, GO and KEGG pathway information for Pristimerin was generated and collected. To further understand the complex relationships among compounds, targets, diseases and pathways, use Cytoscape 3.3 to build and analyze network.

\section{Results}

3.1 ADME-Related Properties of Pristimerin TCMSP provides information on important ADME-related properties such as human oral bioavailability (OB), drug-likeness (DL), Caco-2 permeability (Caco-2), blood-brain barrier (BBB) permeability and Lipinski's rule of five (MW, AlogP, TPSA, Hdon, Hacc)[13]. In-depth study of ADME related characteristics of ASS by TCMSP (Table 1). Notably, the DL of Pristimerin was calculated to be 0.77 (Table 1).

Table 1. Pharmacological and molecular properties of Pristimerin.

\begin{tabular}{cccccccccccc}
\hline NAME & MW & AlogP & Hdon & Hacc & OB(\%) & Caco-2 & BBB & DL & FASA- & TPSA & RBN \\
\hline PM & 464.70 & 5.54 & 1 & 4 & 19.27 & 0.70 & 0.07 & 0.77 & 0.30 & 63.60 & 2 \\
\hline
\end{tabular}

3.2 Recognition of Potential Targets As showed in Figure 1B, the top 300 potential protein targets of Pristimerin were predicted from all 1961 pharmacophore models obtained using 
PharmMapper, as well as the potential 93 targets identified using Coremine. Finally, to improve the specificity, 13 potentially overlapping proteins identified in both sets of results were selected for further study (Table 2).

Table 2. Putative targets of Pristimerin identified by PharmMapper and Coremine

\begin{tabular}{|c|c|c|c|}
\hline Rank & PDB ID & Gene Name & Target Name \\
\hline 1 & 2PQF & PARP1 & poly(ADP-ribose) polymerase 1 \\
\hline 2 & $1 \mathrm{~T} 2 \mathrm{R}$ & AGO2 & argonaute 2 \\
\hline 3 & 2E19 & ZEB1 & zinc finger E-box binding homeobox 1 \\
\hline 4 & $1 \mathrm{SP} 3$ & CYCS & cytochrome c, somatic \\
\hline 5 & $3 \mathrm{~F} 3 \mathrm{P}$ & NUP62 & nucleoporin 62 \\
\hline 6 & $1 W W U$ & EGFR & epidermal growth factor receptor \\
\hline 7 & $2 \mathrm{PPH}$ & MAPK3 & mitogen-activated protein kinase 3 \\
\hline 8 & $1 \mathrm{MYP}$ & MPO & myeloperoxidase \\
\hline 9 & $2 \mathrm{PFF}$ & FASN & fatty acid synthase \\
\hline 10 & $1 \mathrm{MJT}$ & NOS2 & nitric oxide synthase 2 \\
\hline 11 & 1GWI & CYP3A4 & cytochrome P450 family 3 subfamily A member 4 \\
\hline 12 & 2ILK & IL10 & interleukin 10 \\
\hline 13 & $3 \mathrm{~F} 4 \mathrm{M}$ & TNF & tumor necrosis factor \\
\hline
\end{tabular}

3.3 Analysis by GeneMANIA It was found that among the 13 targets and their interacting proteins, 58.93\% shared the same pathway, 20.78\% displayed similar co-expression characteristics. Other results including pathways, physical interactions, and co-localization as shown in Figure 2. 


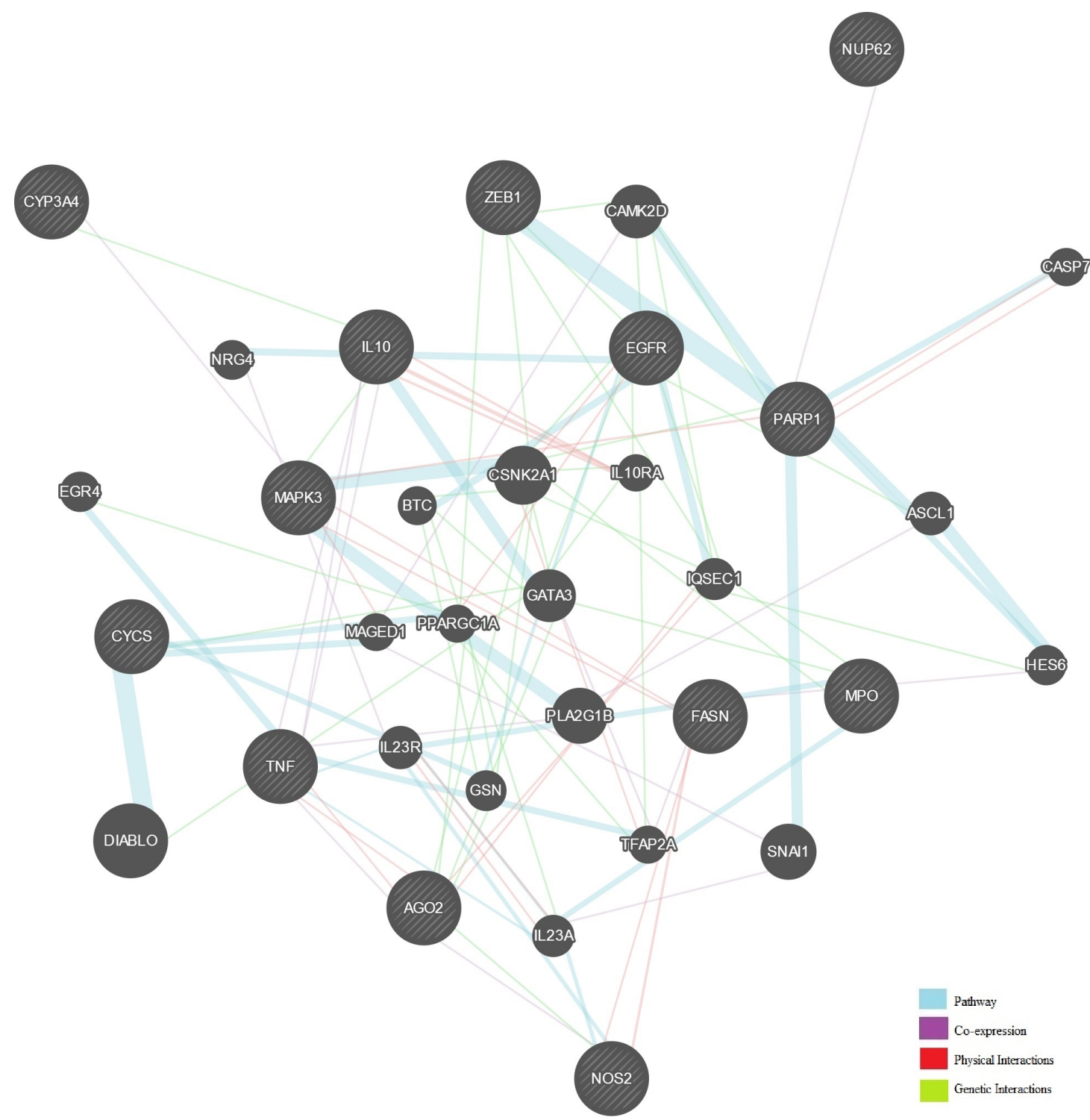

Figure 2. Network of potential Pristimerin targets. Black protein nodes indicate target proteins, and different connecting colours represent different correlations. Functional association of targets was analysed using GeneMANIA.

3.4 GO and Pathway Analysis, and Network Construction To further investigate the 13 identified targets, analysis of interaction network regulation was performed using PANTHER (http://pantherdb.org). As shown in Figure 3, 95\% focus on the biological process. In particular, the top six enriched in negative regulation of growth of symbiont in host (GO:0044130), regulation of chronic inflammatory response to antigenic stimulus (GO:0002874), receptor biosynthetic process (GO:0032800), negative regulation of cytokine secretion involved in immune response (GO:0002740), cellular response to amino acid stimulus (GO:0071230), and endothelial cell apoptotic process (GO:0072577). 


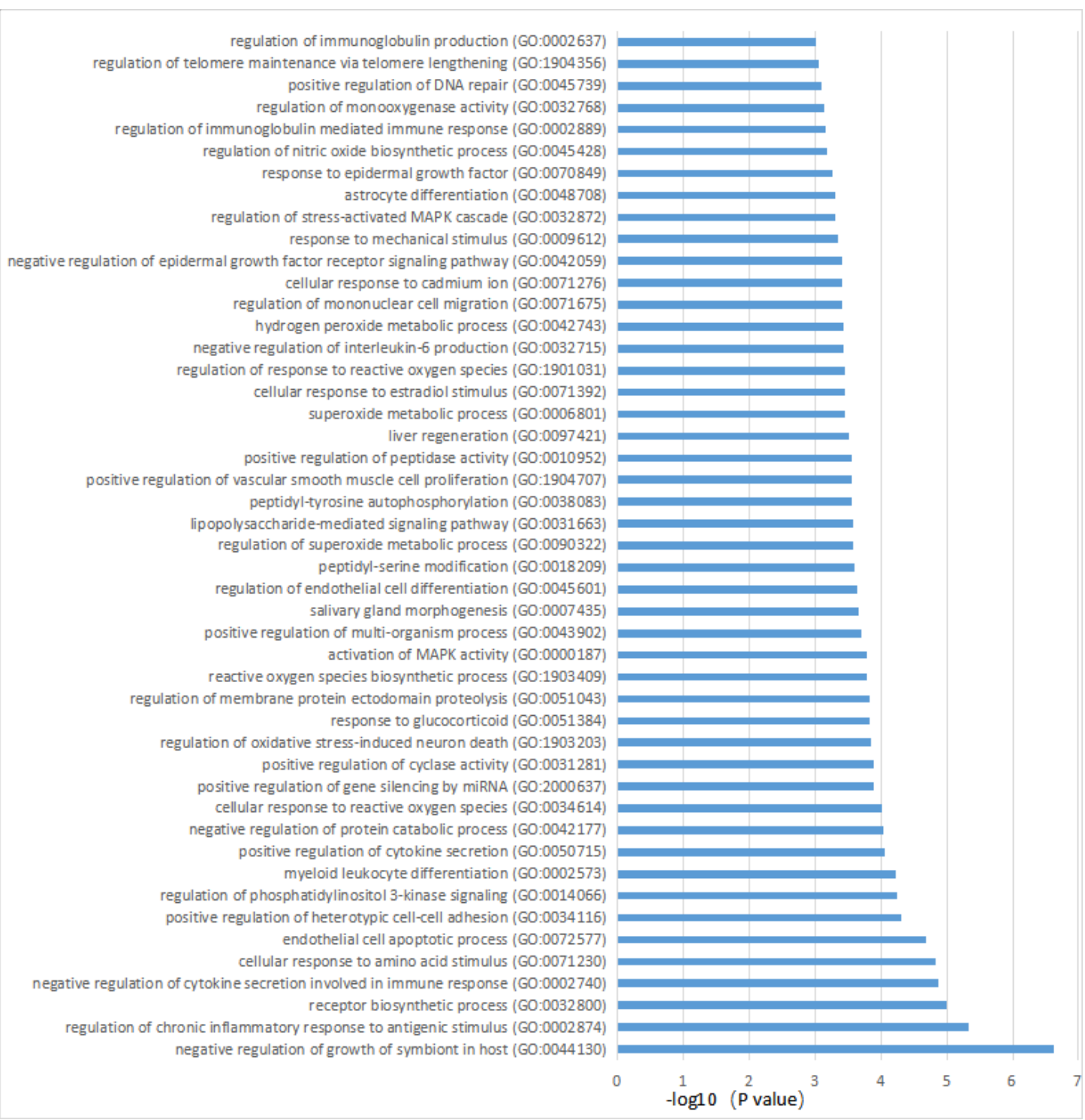

Figure 3. Gene Ontology (GO) analysis of targets. The y-axis shows significantly enriched Biological Process categories of the targets, and the $x$-axis shows the enrichment scores of these terms ( $\mathrm{p}$ value $<0.05)$.

Based on target fishing and pathway analysis, an entire network was built by using Cytoscape3.0. As shown in Figure 4, the interaction network has 47 nodes and 113 edges. The red octagons, green triangles, blue ellips and yellow diamonds correspond to pristimerin, target proteins, diseases, and pathways, respectively. 


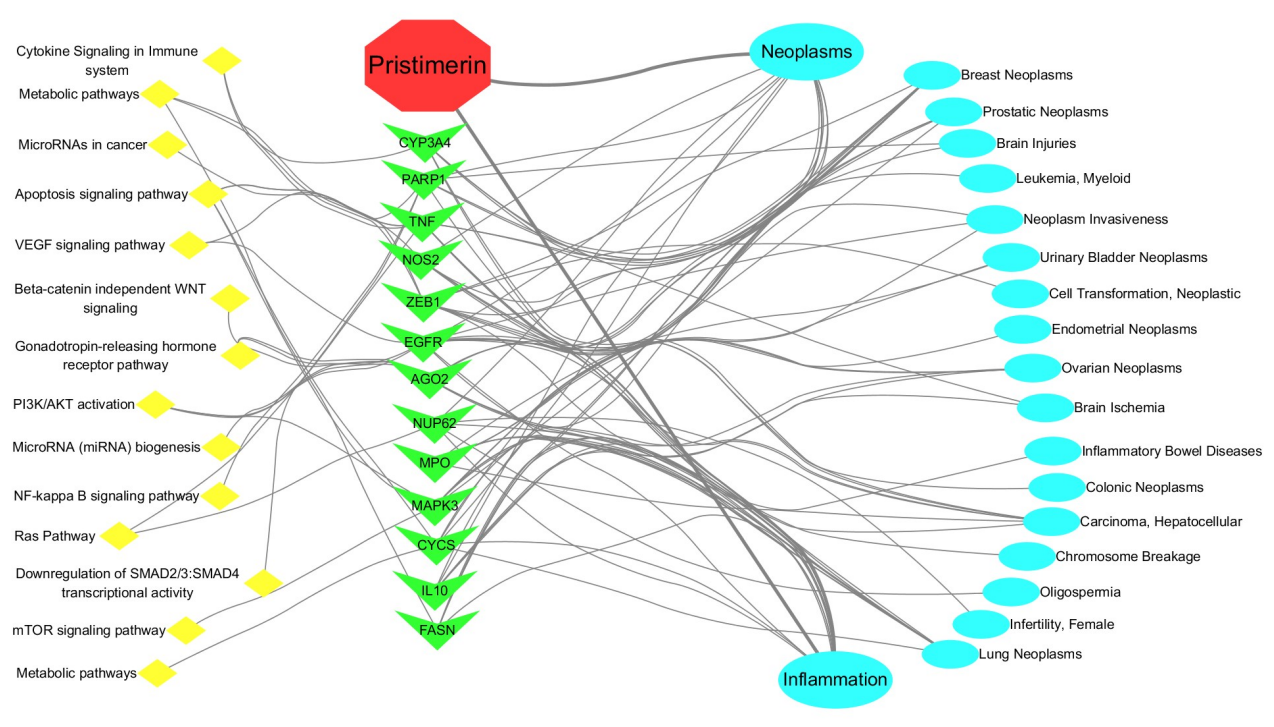

Figure 4. Pristimerin-target-pathway network. Red octagon $=$ pristimerin, green triangle $=$ target proteins, blue ellips= disease, yellow diamond $=$ pathway .

\section{Discussion}

The late-stage failures in drug development often contributed to poor pharmacokinetics and toxicity. In silico approaches can improve our ability to predict and model pharmacokinetic, metabolic and toxicity endpoints, thereby streamlining and accelerating the drug discovery process[18]. In recent years, natural anticancer drugs have received much attention, including Yondelis (trabectedin), a natural compound from the tunicate, Ecteinascidia turbinata, which has been used to treat soft-tissue sarcoma[19, 20]. Pristimerin is extracted as an active ingredient of traditional medicinal plants. Some studies have shown that Pristimerin can inhibit tumor angiogenesis and the development of drug resistance[21, 22].

The molecular weight $(\mathrm{MW})<500$ daltons $(\mathrm{Da})$, the calculated $\log \mathrm{P}(\mathrm{C} \log \mathrm{P})<5$ (or MlogP $>4.15)$, number of hydrogen-bond donors $<5$ and number of hydrogen-bond acceptors $<10$, should be considered for compounds and can identify several critical properties with oral delivery, which were proposed by Lipinski as "rule of five" [23] . Now, the "rule of five" is commonly referred to as a "druglike" measure and guideline in drug. As listed in Table 1, Pristimerin's properties meet the requirements, which means Pristimerin is a good candidate for drug discovery. The concept of DL, established from analysis of physiochemical properties or/and structural characteristics of existing small molecule drugs and/or drug candidates, has been widely used to filter out compounds with undesirable properties, especially those with poor ADMET-related profiles[24]. There are more and more concerns to Natural products with inherently good biological properties. According to reports, in the pharmacological analysis of traditional Chinese medicine systems, the average DL value of drug library compounds $\geq 0.18$ is the standard for screening biologically active compounds[13]. As shown in Table 1, the DL value of Pristinrin was calculated by TCMSP to be 0.77 , which is higher than the average value, thus indicating that Prisminrin may be a promising drug.

Target identification is the first step in drug discovery, and an increasing number of drugs or active compounds have been shown to target multiple proteins. Various in silico target fishing 
methods have been designed and are now widely used for this purpose[25]. As shown in Table 2, 13 putative targets of Pristimerin were screened using computational tools.

Tumor growth has been shown to be a loss of epithelial features and an increase in mesenchymal phenotype procession, known as epithelial-to-mesenchymal transition (EMT)[26], which can be activated by hypoxia-induced HIF-1 signaling[27], and plays a key role in tumor cell metastasis[28]. . Hypoxia also provides clues for tumor cells to maintain stem cell status and may help drive the link between EMT and CSC (cancer stem cells)[29]. Pristimerin is a quinonemethide triterpenoid with the potential of a promising anticancer agent and induces apoptosis[3]. Recent studies reported that Pristimerin not only can suppress HIF-1 $\alpha$ and hypoxia-induced metastasis[30], but also can suppress the hypoxia-induced HIF-1 $\alpha$ accumulation[31] in prostate cancer PC-3 cells. In another word, Pristimerin can inhibit the stimulating effect of hypoxia on cancer cell proliferation and invasion, , suppress stem cell characteristics, and even reduced levels of the predominant mesenchymal phenotype, which is over-expressed in a variety of tumor types and associated with increased metastases and poor prognosis. It has also be confirmed that Pristimerin had the potential value of suppressing colon tumorigenesis. Pristimerin administration can decrease inflammation and proliferation induced by AOM/DSS in colon tissue and induced apoptosis and regulated the AKT/FOXO3a signaling pathway[32].

Cell survival and death are regulated by cell growth and apoptosis signals, and deregulation of either process can lead to cancer[33]. Thus, the induction of apoptosis is one mechanism by which antitumor drugs can kill cancer cells[34]. Pristimerin induces cell death via various mechanisms such as caspase activation, changes in mitochondrial membrane potential, and inhibition of anti-apoptotic factors, including nuclear factor kappa-light-chain-enhancer of activated B cells (NF- $\kappa \mathrm{B})$ and Akt[35].

Apoptosis is induced as a result of caspase activation[36]. There are two major caspase cascades: the intrinsic (mitochondrial) pathway that activates caspases 9 and 3 via cytochrome $\mathrm{C}$ release from mitochondria, and the extrinsic (death receptor) pathway that activates caspases 8 and 10 through receptors (so-called "death receptor") present on the cell membrane surface[37]. In a recently study, Pristimerin induced elevation of caspase 3 and activation of upstream caspases 8 and 9[38]. When Pristimerin induced apoptosis, it was in a dose-dependent manner.

$N F-\kappa B$ is the most important transcription factor involved in inflammation induction, and Pristimerin decreased its expression[39, 40]. It was revealed that Pristimerin, which has long been used as an anti-inflammatory drug, had anti-inflammatory effects by inhibiting NF- $\kappa \mathrm{B}$, which is not only associated with inflammation but also inhibits apoptosis[41]. Firstly, pristimerin inhibited activation of IKK that could be mediated through suppression of upstream kinases such as Akt and MAPK[42, 43]. Secondly, pristimerin inhibited ІкВ $\alpha$ phosphorylation and degradation in a dose and time dependent manners, which eventually leads to the inhibition of phosphorylation and nuclear translocation and DNA binding activities of NF- $\mathrm{KB}$ p65 subunit. These inhibitory effects of pristimerin on NF- $\mathrm{KB}$ activity also reflected by the suppression of NF-KB-dependent genes expression, including Bcl-2, Bcl-xL and cyclin D1[43, 44]. Furthermore, pristimerin-induced inhibition of NF-KB activation was detected a lot of cancer cell lines, including multiple myeloma tumors [5], and pancreatic cancer cells[45].

Activation of the PI3K/Akt/mTOR pathway is one of the drivers of cancer cell growth, and is closely involved in cancer initiation, progression, and treatment resistance. Thus, it is considered an important target in cancer treatment[46]. A currently study showed that Pristimerin aimed at 
suppressing PI3K/Akt pathway, and inhibitors of mTOR are already used in clinical practice[47]. Using Pristimerin can reduce the expression of phosphorylated Akt and mTOR in tumor, which indicative of activation, suggesting that Pristimerin inhibits the growth of cancer cells by inhibiting the Akt/mTOR pathway. The Akt/mTOR pathway and NF- $\kappa B$ cross-talk and induce apoptosis[47]. A comprehensive evaluation of Pristimerin toxicity is yet to reported that no significant be undertaken. Yousef et al[48] change in body weight was observed after treatment of mice with $1 \mathrm{mg} / \mathrm{kg}$ Pristimerin every other day, and minimal toxicity has reported in another study[49]. Pristimerin-associated bone marrow suppression has been reported[50]. Though there were already some investigation of Pristimerin, how and which miRNA can it reactive with is still not clear, and the mechanism of the Pristimerin do with autoimmune diseases is unknown.

\section{Conclusions}

In this study, the DL of Pristimerin was evaluated by TCMSP, and potential targets were chosen for further investigation identified by both PharmMapper and Coremine. The results suggested that Pristimerin may be a good drug candidate, the 13 potential target proteins were identified that are associated with various pharmacological activities. In addition, GO and pathway analysis were performed to construct a drug-target-disease association network. These results indicated that Pristimerin has variety of functions, including anticancer and inflammation activity, even in the autoimmune diseases is still not sure. Although further research is needed to determine the precise interactions, this study provides a systematic and visual overview of possible Pristimerin molecular mechanisms and signaling pathways.

Author Contributions: Writing-review and editing, Zaiyu Li; writing-original draft preparation and formal analysis, Cong $\mathrm{Hu}$; investigation, $\mathrm{Yu}$ Zhen; ethodology and software, Bo Pang; funding acquisition, Huanfa Yi; project administration, Xianglin Chen.

Funding: The research is supported by grant from National Nature Science Foundation of China (81671592), National Nature Science Foundation of China(81501279), Science and Technology Department of Jilin Province: 20180101110JC.

Conflicts of Interest: The authors declare no conflict of interest. 


\section{Reference}

1. Brinker, A. M.; Ma, J.; Lipsky, P. E.; Raskin, I., Medicinal Chemistry and Pharmacology of Genus Tripterygium (Celastraceae). PHYTOCHEMISTRY/2.779 2007, 68, (6), 732-766.

2. Yousef, B. A.; Hassan, H. M.; Zhang, L. Y.; Jiang, Z. Z., Pristimerin exhibits in vitro and in vivo anticancer activities through inhibition of nuclear factor-small ka, CyrillicB signaling pathway in colorectal cancer cells. PHYTOMEDICINE/2.937 2018, 40, 140-147.

3. Liu, Y. B.; Gao, X.; Deeb, D.; Arbab, A. S.; Gautam, S. C., Pristimerin Induces Apoptosis in Prostate Cancer Cells by Down-regulating Bcl-2 through ROS-dependent Ubiquitin-proteasomal Degradation Pathway. Journal of Carcinogenesis \& Mutagenesis 2013, Suppl 6, (3), 005.

4. Wu, C. C.; Chan, M. L.; Chen, W. Y.; Tsai, C. Y.; Chang, F. R.; Wu, Y. C., Pristimerin induces caspase-dependent apoptosis in MDA-MB-231 cells via direct effects on mitochondria. MOL CANCER THER/5.579 2005, 4, (8), 1277.

5. Tiedemann, R. E.; Schmidt, J.; Keats, J. J.; Shi, C. X.; Zhu, Y. X.; Palmer, S. E.; Mao, X.; Schimmer, A. D.; Stewart, A. K., Identification of a potent natural triterpenoid inhibitor of proteosome chymotrypsin-like activity and NF-kappaB with antimyeloma activity in vitro and in vivo. BLOOD/11.841 2009, 113, (17), 4027-4037.

6. Xie, G.; Yu, X.; Liang, H.; Chen, J.; Tang, X.; Wu, S.; Liao, C., Pristimerin overcomes adriamycin resistance in breast cancer cells through suppressing Akt signaling. ONCOL LETT/1.482 2016, 11, (5), 3111-3116.

7. Yousef, B. A.; Hassan, H. M.; Zhang, L. Y.; Jiang, Z. Z., Anticancer Potential and Molecular Targets of Pristimerin: A Mini- Review. CURR CANCER DRUG TAR/3.707 2017, 17, (2), -.

8. Chiang, K. C.; Tsui, K. H.; Chung, L. C.; Yeh, C. N.; Chen, W. T.; Chang, P. L.; Juang, H. H., Celastrol Blocks Interleukin-6 Gene Expression via Downregulation of NF- $\kappa$ B in Prostate Carcinoma Cells. PLOS ONE/3.057 2014, 9, (3), e93151.

9. Shishodia, S.; Majumdar, S.; Banerjee, S.; Aggarwal, B. B., Ursolic acid inhibits nuclear factor-kappaB activation induced by carcinogenic agents through suppression of IkappaBalpha kinase and p65 phosphorylation: correlation with down-regulation of cyclooxygenase 2, matrix metalloproteinase 9 , and cyclin D1. CANCER RES/8.556 2003, 63, (15), 4375-4383.

10. Takada, Y.; Aggarwal, B. B., Betulinic Acid Suppresses Carcinogen-Induced NF-кB Activation Through Inhibition of I $\kappa \mathrm{B} \alpha$ Kinase and p65 Phosphorylation: Abrogation of Cyclooxygenase-2 and Matrix Metalloprotease-9. J IMMUNOL/4.985 2003, 171, (6), 3278-3286.

11. Dong, C.; Xu, C.; Liu, H.; Xu, S.; Gao, Y.; Peng, J., Absorption and metabolism characteristics of pristimerin as determined by a sensitive and reliable LC-MS/MS method. FITOTERAPIA/2.408 2015, $106,62-67$

12. Gao, X.; Zhang, Y.; Wang, Y.; Zhang, Y.; Wang, Y.; Liu, S.; Gao, X., Influence of verapamil on pharmacokinetics of pristimerin in rats. BIOMED CHROMATOGR/1.729 2016, 30, (6), 802-809.

13. Ru, J.; Li, P.; Wang, J.; Zhou, W.; Li, B.; Huang, C.; Li, P.; Guo, Z.; Tao, W.; Yang, Y., TCMSP: a database of systems pharmacology for drug discovery from herbal medicines. Journal of Cheminformatics,6,1(2014-04-16) 2014, 6, (1), 13.

14. Liu, X.; Ouyang, S.; Yu, B.; Liu, Y.; Huang, K.; Gong, J.; Zheng, S.; Li, Z.; Li, H.; Jiang, H. In PharmMapper server: A Web server for potential drug target identification using pharmacophore mapping approach, the 5 th international conference of molecular simulations and applied informatics technologies, 2015; p W609.

15. Chu, H.; Zhou, X.; Liu, G.; Lv, M.; Zhou, X.; Wang, Y.; Liu, L.; Li, X.; Sun, P.; Zhu, Y. In Network-based 
detection of disease modules and potential drug targets in intractable epilepsy, International Conference on Systems Biology, 2014; pp 132-140.

16. Warde-Farley, D.; Donaldson, S. L.; Comes, O.; Zuberi, K.; Badrawi, R.; Chao, P.; Franz, M.; Grouios, C.; Kazi, F.; Lopes, C. T.; Maitland, A.; Mostafavi, S.; Montojo, J.; Shao, Q.; Wright, G.; Bader, G. D.; Morris, Q., The GeneMANIA prediction server: biological network integration for gene prioritization and predicting gene function. NUCLEIC ACIDS RES/9.202 2010, 38, (Web Server issue), W214-20.

17. Mi, H.; Muruganujan, A.; Thomas, P. D., PANTHER in 2013: modeling the evolution of gene function, and other gene attributes, in the context of phylogenetic trees. NUCLEIC ACIDS RES/9.202 2013, 41, (Database issue), 377-86.

18. Van, d. W. H.; Gifford, E., ADMET in silico modelling: towards prediction paradise? NAT REV DRUG DISCOV/47.12 2003, 2, (3), 192-204.

19. Newman, D. J.; Cragg, G. M., Natural Products as Sources of New Drugs from 1981 to 2014. J NAT PROD/3.662 2016, 79, (3), 629.

20. De, S. R.; Marrari, A.; Santoro, A., Trabectedin for the treatment of soft tissue sarcomas. EXPERT OPIN PHARMACO/3.543 2016, 17, (11), 1569.

21. Mu, X.; Shi, W.; Sun, L.; Li, H.; Jiang, Z.; Zhang, L., Pristimerin, a triterpenoid, inhibits tumor angiogenesis by targeting VEGFR2 activation. MOLECULES/2.465 2012, 17, (6), 6854.

22. Xie, G.; Yu, X.; Liang, H.; Chen, J.; Tang, X.; Wu, S.; Liao, C., Pristimerin overcomes adriamycin resistance in breast cancer cells through suppressing Akt signaling. ONCOL LETT/1.482 2016, 11, (5), 3111-3116.

23. Leeson, P. D., Molecular Inflation, Attrition and the Rule of Five. ADV DRUG DELIVER REV/15.606 2016, 101, 22-33.

24. Sheng, T.; Junmei, W.; Youyong, L.; Dan, L.; Lei, X.; Tingjun, H., The application of in silico drug-likeness predictions in pharmaceutical research. ADV DRUG DELIVER REV/15.606 2015, 86, 2-10.

25. Ceretomassagué, A.; Ojeda, M. J.; Valls, C.; Mulero, M.; Pujadas, G.; Garciavallve, S., Tools for in silico target fishing. METHODS/3.503 2015, 71, 98-103.

26. Ben, D.; Tropé, C. G.; Reuven, R., Epithelial-Mesenchymal Transition in Ovarian Carcinoma. Frontiers in Oncology 2012, 2, 33.

27. Higgins, D. F.; Kimura, K.; Bernhardt, W. M.; Shrimanker, N.; Akai, Y.; Hohenstein, B.; Saito, Y.; Johnson, R. S.; Kretzler, M.; Cohen, C. D., Hypoxia promotes fibrogenesis in vivo via HIF-1 stimulation of epithelial-to-mesenchymal transition. J CLIN INVEST/12.575 2007, 117, (12), 3810.

28. Hanahan, D.; Weinberg, R. A., Hallmarks of cancer: the next generation. CELL/28.71 2011, 144, 646-674.

29. Marieegyptienne, D. T.; Lohse, I.; Hill, R. P., Cancer stem cells, the epithelial to mesenchymal transition (EMT) and radioresistance: potential role of hypoxia. CANCER LETT/5.992 2013, 341, (1), 63-72.

30. Zuo, J.; Guo, Y.; Peng, X.; Tang, Y.; Zhang, X.; He, P.; Li, S.; Wa, Q.; Li, J.; Huang, S.; Xu, D., Inhibitory action of pristimerin on hypoxiamediated metastasis involves stem cell characteristics and EMT in PC-3 prostate cancer cells. ONCOL REP/2.486 2015, 33, (3), 1388-94.

31. Lee, S. O.; Kim, J. S.; Lee, M. S.; Lee, H. J., Anti-cancer effect of pristimerin by inhibition of HIF-1alpha involves the SPHK-1 pathway in hypoxic prostate cancer cells. BMC CANCER/3.265 2016, 16, 701.

32. Park, J. H.; Kim, J. K., Pristimerin, a naturally occurring triterpenoid, attenuates tumorigenesis in experimental colitis-associated colon cancer. PHYTOMEDICINE/2.937 2018, 42, 164-171.

33. Hanahan, D.; Weinberg, R. A., The hallmark of cancer. CELL/28.71 2000, 100, 57-71.

34. Dasari, S.; Tchounwou, P. B., Cisplatin in cancer therapy: Molecular mechanisms of action. EUR J 
PHARMACOL/2.73 2014, 740, 364-378.

35. Gao, X.; Liu, Y.; Deeb, D.; Arbab, A. S.; Gautam, S. C., Anticancer activity of pristimerin in ovarian carcinoma cells is mediated through the inhibition of prosurvival Akt/NF- $\kappa \mathrm{B} / \mathrm{mTOR}$ Signaling. Journal of Experimental Therapeutics \& Oncology 2014, 10, (4), 275-83.

36. Denault, J. B.; Salvesen, G. S., Caspases: keys in the ignition of cell death. Cheminform 2002, 34, (12), 4489-500.

37. Wong, R. S., Apoptosis in cancer: from pathogenesis to treatment. Journal of Experimental \& Clinical Cancer Research Cr 2011, 30, (1), 87-87.

38. Mori, Y.; Shirai, T.; Terauchi, R.; Tsuchida, S.; Mizoshiri, N.; Hayashi, D.; Arai, Y.; Kishida, T.; Mazda, O.; Kubo, T., Antitumor effects of pristimerin on human osteosarcoma cells in vitro and in vivo. Onco Targets Ther 2017, 10, 5703-5710.

39. Brasier, A. R., The NF-kappaB regulatory network. CARDIOVASC TOXICOL/2.063 2005, 6, (2), 111-130.

40. Dalgleish, A. G.; Ken, O.; Byrne, Inflammation and Cancer. NATURE/38.138 2002, 420, (6917), 860-867.

41. Karin, M., Nuclear factor-kappaB in cancer development and progression. NATURE/38.138 2006, 441, (7092), 431-436.

42. Yan, Y.; Bai, J.; Xie, Y.; Jiezhong, Y. U.; Cungen, M. A., The triterpenoid pristimerin induces U87 glioma cell apoptosis through reactive oxygen species-mediated mitochondrial dysfunction. ONCOL LETT/1.482 2013, 5, (1), 242-248.

43. Yousef, B. A.; Guerram, M.; Hassan, H. M.; Hamdi, A. M.; Zhang, L. Y.; Jiang, Z. Z., Pristimerin demonstrates anticancer potential in colorectal cancer cells by inducing G1 phase arrest and apoptosis and suppressing various pro-survival signaling proteins. ONCOL REP/2.486 2016, 35, (2), 1091.

44. Wang, Y.; Zhou, Y.; Zhou, H.; Jia, G.; Liu, J.; Han, B.; Cheng, Z.; Jiang, H.; Pan, S.; Sun, B., Pristimerin causes G1 arrest, induces apoptosis, and enhances the chemosensitivity to gemcitabine in pancreatic cancer cells. PLOS ONE/3.057 2012, 7, (8), e43826.

45. Deeb, D.; Gao, X.; Liu, Y. B.; Pindolia, K.; Gautam, S. C., Pristimerin, a quinonemethide triterpenoid, induces apoptosis in pancreatic cancer cells through the inhibition of pro-survival Akt/NF- $\mathrm{kB} / \mathrm{mTOR}$ signaling proteins and anti-apoptotic Bcl-2. INT J ONCOL/3.018 2014, 44, (5), 1707-1715.

46. Jr, P. J.; Janku, F., Molecular targets for cancer therapy in the PI3K/AKT/mTOR pathway. PHARMACOL THERAPEUT/11 2014, 142, (2), 164-175.

47. Brown, J. S.; Banerji, U., Maximising the potential of AKT inhibitors as anti-cancer treatments. PHARMACOL THERAPEUT/11 2016, 172, 101.

48. Yousef, B. A.; Hassan, H. M.; Guerram, M.; Hamdi, A. M.; Wang, B.; Zhang, L. Y.; Jiang, Z. Z., Pristimerin inhibits proliferation, migration and invasion, and induces apoptosis in HCT-116 colorectal cancer cells. BIOMED PHARMACOTHER/2.326 2016, 79, 112-119.

49. Wang, L. N.; Wang, Y.; Lu, Y.; Yin, Z. F.; Zhang, Y. H.; Aslanidi, G. V.; Srivastava, A., Pristimerin enhances recombinant adeno-associated virus vector-mediated transgene expression in human cell lines in vitro and murine hepatocytes in vivo. Journal of Integrative Medicine 2014, 12, (1), 20-34.

50. Lu, Z.; Jin, Y.; Chen, C.; Li, J.; Cao, Q.; Pan, J., Pristimerin induces apoptosis in imatinib-resistant chronic myelogenous leukemia cells harboring T315I mutation by blocking NF- $\kappa$ B signaling and depleting Bcr-Abl. MOL CANCER/5.888 2010, 9, (1), 112. 